\title{
A Wireless Real-Time Monitoring Node of the Physiological Signals for Unrestrained Dairy Cattle Using Wireless Sensor Network
}

\author{
Xihai Zhang, Changli Zhang*, Junlong Fang, and Yongcun Fan \\ Engineering college, Northeast Agriculture University, Harbin, \\ Heilongjiang Province, P.R. China 150030, \\ Tel.: +86-451-55191146 \\ xhzhang@neau .edu.cn
}

\begin{abstract}
A newly developed smart sensor node that can monitor physiological signals for unrestrained dairy cattle is designed through modular design and its advantages are compact structure and small volume. This sensor node is based on a MSP430F133 micro-controller; the digital sensor includes temperature sensor (DS18B20-America) and vibration-displacement sensor (DN series China); transmission of the digital data uses the nRF903. The results show that this node can collect physiological signals for unrestrained dairy cattle and then send it to upper network node. This research can provide better hardware platform for further researching the communication protocols of wireless sensor networks.
\end{abstract}

Keywords: dairy cattle, physiological signals, wireless sensor network, ZigBee.

\section{Introduction}

The physiological signals always reflect the presence status of dairy cattle, such as increased activity amount that maybe indicate estrus or abnormal body temperature that maybe imply malfunction or disease. However, at present, the monitoring of physiological signals for dairy cattle almost depends on dairyman's observation and judgment by experience, which is not quite accurate and timely. Therefore, the accurate and timely monitoring system of physiological signals should be established for allowing us to better understand of cattle status.

Some techniques have been used successfully for recording and storing physiological signals in unrestrained animals, often based on the mounting of some type of tape recorder or data logger package on the animal in Ref. (Hahn et al., 1990; Feddes et al., 1993; Eigenberg et al., 2002). In a few cases, it is difficult to realize wireless real-time monitoring signal by these techniques (Lowea et al., 2007).

A wireless sensor network is very feasible for on-line data collection tasks and animal monitoring, which can greatly extend our ability to monitor the physiological signals of unrestrained livestock from remote locations. However, there are a few

* Corresponding author. 
obstacles that must be overcome before a general purpose sensor network can be developed. These obstacles arise from the limited energy, computational power, and communication recourses available to the sensors in the network (Silva et al., 2005).

Based on these considerations, this paper presents a wireless sensor network node for real-time monitoring the estrus and body temperature of unrestrained dairy cattle. This system consists of cattle module, which is responsible for the amplification and transmission of body data. The system adopts the ZigBee wireless technology, which enables long battery lifetime and offers the opportunity to build up complex wireless networks of sensors.

\section{Hardware Design}

The system consists of two main modules: the first module is responsible for the amplification and transmission of acquired cattle physiological signals, and the second module receives data and connects the system to a host. The system architecture is shown in Fig. 1.

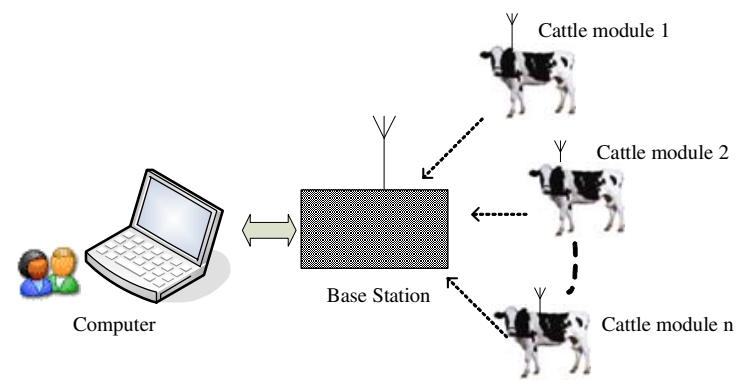

Fig. 1. System architecture

The cattle module is placed on the cattle, which measures physiological signals through sensors, digitalizes data, and transmits it to the base module. This module consists of three main parts. (1) digital sensor includes temperature and vibrationdisplacement sensor; (2) digital amplification; (3) microprocessor; (4) transmission of the digital data. Those modules use an I2C bus that can be connected to the different digital sensor instruments. The hardware of node is shown in Fig.2.

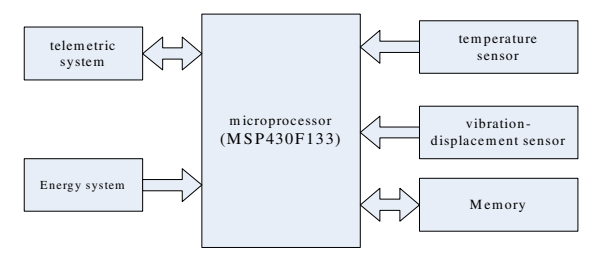

Fig. 2. The composing frame of data collector 


\subsection{Digital Sensor}

\subsubsection{Temperature Sensor}

The DS18B20 communicates over a 1-Wire bus that by definition requires only one data line for communication with a central microprocessor. It has an operating temperature range of $-55^{\circ} \mathrm{C}$ to $+125^{\circ} \mathrm{C}$ and is accurate to $\pm 0.5^{\circ} \mathrm{C}$ over the range of $10^{\circ} \mathrm{C}$ to $+85^{\circ} \mathrm{C}$. In addition, the DS18B20 can derive power directly from the data line, eliminating the need for an external power supply (Wang et al., 2007).

The 1-Wire bus system uses a single bus master to control one or more slave devices. The DS18B20 is always a slave. When there is only one slave on the bus, the system is referred to as a "single-drop" system; the system is "multidrop" if there are multiple slaves on the bus.

The communication circuit between 18B20 and MSP430F133 is shown in Fig.3. The VDD is connected to external supply. The 1-Wire bus requires an external pull up resistor of approximately $5 \mathrm{k} \Omega$; thus, the idle state for the 1 -Wire bus is high. If for any reason, a transaction needs to be suspended.

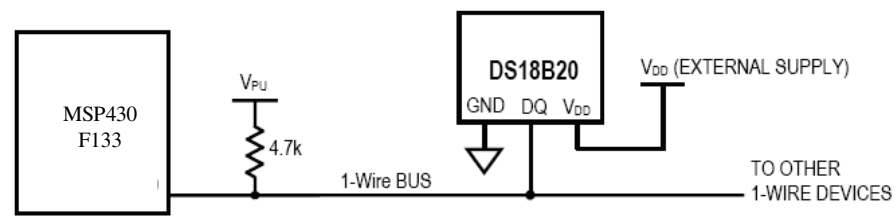

Fig. 3. The interface circuit between $18 \mathrm{~B} 20$ and $\mathrm{MCU}$

\subsubsection{Vibration-Displacement Sensor}

According to monitor requirement, we use vibration-displacement sensor (DN seriesChina). It has high detection sensitivity and good anti-interference ability. DN series sensor is not mechanical fatigue, false trigger, and so on for working at solid detection mode. The main parameter is as follows:

(1) Sensitivities: 0.19;

(2) Volume: $0.8 \times 2 \times 2.2 \mathrm{~cm}$, it is shown in Fig. 4 .

(3) Test direction: omnidirectional;

(4) Working temperature:- $30^{\circ} \mathrm{C} \sim 65^{\circ} \mathrm{C}$

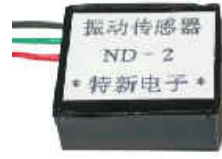

ND-2

Fig. 4. ND-2

\subsection{Microprocessor}

Microprocessor uses MSP430F133. The MSP430F133 captures signals of temperature and vibration-displacement, convert them to digital values and process and transmit the data to a base module. The timers make the configurations ideal for industrial control applications such as ripple counters, digital motor control, EEmeters, hand-held meters, etc. The hardware multiplier enhances the performance and offers a broad code and hardware-compatible family solution. 


\subsection{Transmission Module}

Transmission Module uses nRF903. The nRF903 is a true single chip multi-channel UHF transceiver designed to operate in the unlicensed $433 \mathrm{MHz}, 868 \mathrm{MHz}$ and 915MHz ISM-/LPRD-bands. Multi-channel operation, excellent receiver selectivity and sensitivity, high bandwidth efficiency and blocking performance make the nRF903 suitable for wireless links where high reliability is a key requirement.

The device features GFSK modulation and demodulation capability at an effective bit rate of $76.8 \mathrm{kbit} / \mathrm{s}$ in $153.6 \mathrm{kHz}$ channel bandwidths. Transmit power can be adjusted to a maximum of $10 \mathrm{dBm}$ which is available for all frequency bands and channels. Antenna interface is differential and suited for low cost PCB-antennas. All necessary configuration data is programmed by a 14-bit configuration word via a SPI.

\section{Design of Node Software}

Node software realizes its functions of data collecting, data processing under the guide idea of modularization. A characteristic of this software is its good utility, expansibility and operating.

\subsection{Temperature Collection}

The transaction sequence for accessing the DS18B20 is as follows:

Step 1. Initialization;

Step 2. ROM Command (followed by any required data exchange);

Step 3. DS18B20 function command.

It is very important to follow above sequence every time the DS18B20 is accessed, as the DS18B20 will not respond if any steps in the sequence are missing or out of order.

\subsection{Programing of nRF903}

Step 1. Initialization : Setting registers in chip, working frequency and transmit power.

Step 2. Entering normal working state: working transceiver conversion control, sending data and state transition.

\subsection{ZigBee-Stack}

The ZigBee protocol is designed to communicate with experimental modules without information loss, using minimum microprocessor program memory space. It is a distributed medium access control protocol that provides robustness against single node failure and supports for flexible topologies, in which nodes are partially connected and not all nodes need to have a direct communication with a host. This process repeats until a maximal interval of data receiving was reached (Sinem et al., 2006).The system structure of ZigBee-stack is shown in Fig.5.

ZigBee-Stack operates in an OSAL operation system. The task in OSAL adds to this system by API, which could realize the multitask mechanism. The OSAL task 


\begin{tabular}{|c|c|c|c|}
\hline \multicolumn{2}{|c|}{ Application Layer } & & \multirow{2}{*}{ 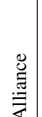 } \\
\hline \multicolumn{2}{|c|}{ Network Layer } & & \\
\hline \multicolumn{2}{|c|}{ MAC } & $\begin{array}{l}+ \\
\stackrel{0}{0}\end{array}$ & 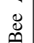 \\
\hline $\begin{array}{l}\text { IEEE } 802.15 .4 \\
868 / 915 \mathrm{MHz} \text { PHY }\end{array}$ & $\begin{array}{c}\text { IEEE } 802.15 .4 \\
\text { 2.4GHz PHY }\end{array}$ & 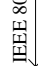 & \\
\hline
\end{tabular}

Fig. 5. ZigBee system structure diagram

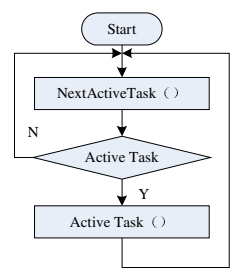

Fig. 6. OSAL task scheduling mechanism

scheduling is shown in Fig.6. NextActiveTask( ) is task event query function what returns the ActiveTask. When we design software, we can decide if executing to corresponding ActiveTask( ) by the value of ActiveTask.

\section{Conclusion}

In this paper, a novel WSN node for monitoring physiological signals in unrestrained cattle was designed, which based on MSP430F133, DS18B20 and nRF903. The sensor node is designed through modular design and its advantage is compact structure and small volume. The node can collect physiological signals for unrestrained dairy cattle and send it to upper network node. This research can provide better hardware platform for further researching the communication protocols of wireless sensor networks. The next work will complete the whole system, including many cattle modules, station module and host (or PDA).

\section{Acknowledgements}

Funding for this research was provided by Heilongjiang youth science and technology special funds project (No. QC2009C18); Innovation Talent Research Fund of Harbin Science and Technology Bureau (2008RFXXN003); Innovation Team Fund of Northeast Agriculture University (CXZ010-1).

\section{References}

Silva, A.C.S., Arce, A.I.C., Souto, S., et al.: A wireless floating base sensor network for physiological responses of livestock. Computers and electronics in agriculture 49, 246-254 (2005)

Sinem, C.E.: Power efficient and delay aware medium access protocol for sensor networks. IEEE Transactions on Mobile Computing 5(7), 920-930 (2006)

Hahn, G.L., Eigenberg, R.A., Nienaber, J.A., et al.: Measuring physiological-responses of animals to environmental stressors using a microcomputer-based portable data logger. J. Anim. Sci. 68, 2658-2665 (1990) 
Lowea, J.C., Abeyesinghea, S.M., Demmersa, T.G.M., et al.: A novel telemetric logging system for recording physiological signals in unrestrained animals. Computers and electronics in agriculture 57, 74-79 (2007)

Feddes, J.J.R., Deshazer, J.A.: Development of a portable microprocessor for measuring selected stress responses of growing pigs. Trans. Am. Soc. Agric. Eng. 36, 201-204 (1993)

Eigenberg, R.A., Brown-Brandl, T., Nienaber, J.A.: Development of a respiration rate monitor for swine. Trans. Am. Soc. Agric. Eng. 45, 1599-1603 (2002)

Wang, W., Zebin, F.: Design of wireless temperature detect based on CC2430. Electronic Engineer 33(8), 78-80 (2007) (in Chinese) 